\title{
Traction/Braking Force Distribution for Optimal Longitudinal Motion During Curve Following
}

\author{
Huei Peng ${ }^{1}$, Jwu-Sheng $\mathrm{Hu}^{2}$
}

\section{SUMMARY}

The optimal tire force distribution to maximize acceleration/deceleration of a four-wheel vehicle during cornering is studied. The objective of this research is to investigate the improvement one can expect from the implementation of different vehicle steering and driving mechanisms. We first identify the upper limit imposed by physical laws by assuming all the four wheels can be individually steered and driven. Practical vehicle configurations such as fourwheel-steering (4WS) and four-wheel-drive (4WD) are then considered. The optimization involves equality and inequality constraints and is solved by nonlinear programming techniques.

\section{Introduction}

The handling and traction/braking characteristics of road vehicles have been greatly improved in the past twenty years through the use of electronic devices. Four-wheel-steering (4WS) systems have been developed to enhance the vehicle maneuverability and stability. The rear wheel steering has been utilized to eliminate vehicle side slip angle, reduce turning radius at low vehicle speed, decouple lateral and yaw motions, and reduce phase lag between vehicle yaw rate and lateral acceleration (e.g. Takiguchi et al. 1986, Sano et al. 1986, Eguchi et al. 1989). On another vein, anti-lock braking systems (ABS) and traction control systems (TCS) have been developed (Peterson et al. 1988, Rittmannsberger 1988, Shiraishi et al. 1989) to prevent wheel lock-up and spinning, which improve the acceleration/deceleration performance as well as directional stability of vehicles especially on slippery roads. Due to the increasing demand on safety,

\footnotetext{
${ }^{1}$ Corresponding author, Assistant Professor, Department of Mechanical Engineering and Applied Mechanics, University of Michigan, Ann Arbor, MI 48109, U.S.A. Tel: 313-936-0352, Fax: 313 747-3170, E-mail: hpeng@umich.edu

2 National Chiao-Tung University, Hsin-Chu, Taiwan, the Republic of China.
} 
performance, and cost reduction, there were many efforts on the integration of various electronic components (Anto 1988; Schilke et al. 1988), especially chassis electronic control systems (Ressler et al. 1988; Kizu et al. 1988) for improved vehicle dynamic response.

Recently, integrated chassis control techniques (e.g., Sato et al. 1992) have been proposed for improved handling and safety. When the vehicle is accelerating/decelerating in a curve, proper distribution of tire forces is important to maintain vehicle stability. Various systems have been proposed to control vehicle behavior by distributing front-rear and left-right braking forces (Nakazato et al., 1989; Kimbrough, 1990; Matsumoto et al., 1992), adjusting rear-wheel steering angle, or both (Yamamoto, 1991). Most of these work was developed from human-assistance viewpoint. The dynamics of the vehicle was compensated for so that it becomes more maneuverable and stable for the human drivers. One popular approach is to interpret the human controlled steering wheel angle as the desired level of vehicle yaw motion, and the control system was designed to follow the yaw motion command as close as possible. The effect of traction force distribution on vehicle lateral dynamics has also been studied (Motoyama et al. 1992). A PD control algorithm was designed to distribute traction torque for improved yaw response.

In this paper, we propose a design approach to determine the optimal tire force distribution for a vehicle when it is following a curve. The curvature of the road and vehicle speed are assumed to be known. The control objective is to achieve maximum longitudinal acceleration/deceleration without compromising the capability of following the curve. Four scenarios based on vehicle configurations are presented. In the first scenario, the steering and traction of all the four wheels are assumed to be individually controllable (eight control inputs). This scenario outlines the performance limit governed by physical laws. In the second scenario, the vehicle is assumed to be 4WS (two steering inputs), and single-wheel-drive (four driving inputs). In the third scenario, the performance of a $4 \mathrm{WS}$ and $4 \mathrm{WD}$ vehicle is investigated. In the last scenario, the steering is assumed to be controlled by a human driver. The traction/braking force of the tires are assumed to be regulated by a control system, which is constrained not to 
generate any yawing moment. This configuration corresponds to the upper limit of human-steered vehicles. Any effort to exceed this limit implies that the operations of the vehicle control system will interfere human steering, which is not desirable from human factors viewpoint. It should be noted that the main purpose of this paper is to investigate the performance limit under different vehicle configurations. Results from this study can be used for the evaluation and fine-tuning of feedback traction/braking control systems. The implementation issues, however, are not addressed in this paper.

\section{Nomenclature}

a: distance from vehicle mass center to the front wheels

b: distance from vehicle mass center to the rear wheels

$F_{x f}$ : traction/braking force of the front axle

$F_{x r}: \quad$ traction/braking force of the rear axle

$F_{x i}: \quad$ traction/braking force of the ith tire, $\mathrm{i}=1 . .4$

$F_{y i}: \quad$ lateral force of the ith tire, $\mathrm{i}=1 . .4$

$F_{z f}: \quad$ normal force of the front axle

$F_{z r}: \quad$ normal force of the rear axle

$F_{z i}: \quad$ normal force of the ith tire, $\mathrm{i}=1 . .4$

$\mathrm{h}$ : height of vehicle mass center

$I_{z}$ : moment of inertia of the vehicle in the $\mathrm{z}$ (yaw) direction

$k_{f}$ : vehicle lateral weight-shift distribution on front wheels $(=0.45)$

$k_{r}$ : vehicle lateral weight-shift distribution on rear wheels $(=0.55)$

$\mathrm{m}$ : mass of the vehicle

$s_{b}$ : track of the vehicle (assumed to be the same for front and rear axles)

V: $\quad$ vehicle longitudinal speed

$\mu: \quad \mathrm{road} /$ tire friction coefficient

$\rho: \quad$ road radius of curvature

\section{Decoupled Traction/Braking Control Design}

In this section, a decoupled vehicle traction/braking control algorithm is presented. The decoupling is in the sense that the traction/braking force 
distribution is calculated without considering vehicle lateral motion. Assuming that the vehicle lateral and yaw motions are small, a bicycle model of the vehicle can be utilized. The normal forces of the front and rear axles can be obtained as follows:

$$
\begin{aligned}
& F_{z f}=\frac{b m g}{a+b}-\frac{\left(F_{x r}+F_{x f}\right) h}{a+b} \\
& F_{z r}=\frac{a m g}{a+b}+\frac{\left(F_{x r}+F_{x f}\right) h}{a+b}
\end{aligned}
$$

These quasi-static equations are obtained from the equilibrium of pitch moment around the vehicle center of gravity. It should be noted that the vehicle roll/pitch motion is assumed to be kept small in this paper. Effects due to large vehicle body motion on tire force properties are thus neglected. Since the lateral tire forces are assumed to be small, tire longitudinal force characteristic can be described by the curve shown in Fig. 1, where the normalized tire longitudinal force increases almost linearly with tire slip ratio $\lambda$ when $\lambda$ is small, and gradually decreases after a peak value, $\mu$, is reached. The traction force transmitted to the tires should be kept less than $\mu F_{z}$ to prevent tire spinning. In other words, the following relationship should be maintained:

$$
\begin{aligned}
& \frac{F_{x r}}{F_{z r}} \leq \mu \\
& \frac{F_{x f}}{F_{z f}} \leq \mu
\end{aligned}
$$

where $\mu$ is the peak value of the normalized longitudinal tire force shown in Fig. 1 , and is assumed to be the same for the front and rear axles.

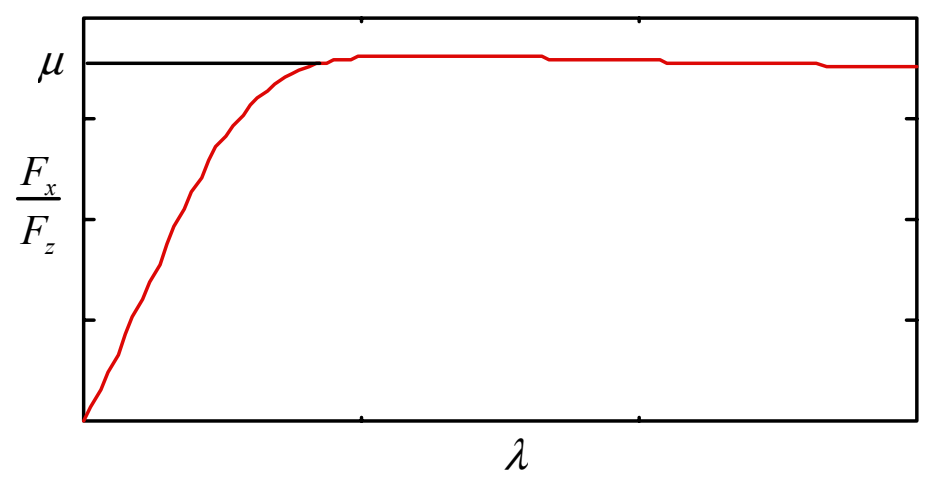

Figure 1: Relationship between tire longitudinal force and tire slip ratio 
Combining equations (1) through (4) and assuming that $\mu h<a+b$, the following equations can be obtained:

$$
\begin{aligned}
& F_{x r} \leq \mu \frac{a m g+h F_{x f}}{a+b-\mu h} \\
& F_{x f} \leq \mu \frac{b m g-h F_{x r}}{a+b+\mu h}
\end{aligned}
$$

Eqs. (5) and (6) are plotted in Figure 2 for different values of $\mu$ using the system parameters listed in Table 1. The wheel traction forces are normalized against the vehicle weight, $m g$. In Figure 2(a), $\mu$ is assumed to be 0.85 . The maximum acceleration that can be achieved by a front wheel drive (FWD) vehicle is a little bit less than $0.4 g$, a rear wheel drive (RWD) vehicle can do about $0.5 \mathrm{~g}$, and a $4 \mathrm{WD}$ vehicle can achieve $0.85 \mathrm{~g}\left(F_{x f}=0.3 \mathrm{mg}, F_{x r}=0.55 \mathrm{mg}\right)$. On a slippery road surface ( $\mu=0.3$ ), the best acceleration that can be achieved by FWD, RWD, and $4 W D$ vehicles are $0.145 g, 0.155 g$, and $0.295 g$, respectively. In other words, the performance of FWD and RWD vehicles are comparable, and 4WD vehicles can double the acceleration. The optimal tire force distribution to achieve the best acceleration response can be obtained from the intersection point of the two lines described by equations (5) and (6):

$$
\frac{F_{x f}}{F_{x r}}=\frac{b-\mu h}{a+\mu h}
$$

Eq.(7) shows that when $\mu$ is large (on normal dry road surface), rear tires should bear more traction force to account for the weight shift effect. When $\mu$ is 0.85 , the force distribution should be $35 / 65$ (front/rear) to achieve maximum vehicle acceleration, and when $\mu$ is 0.3 , the distribution becomes $46 / 54$ (front/rear).
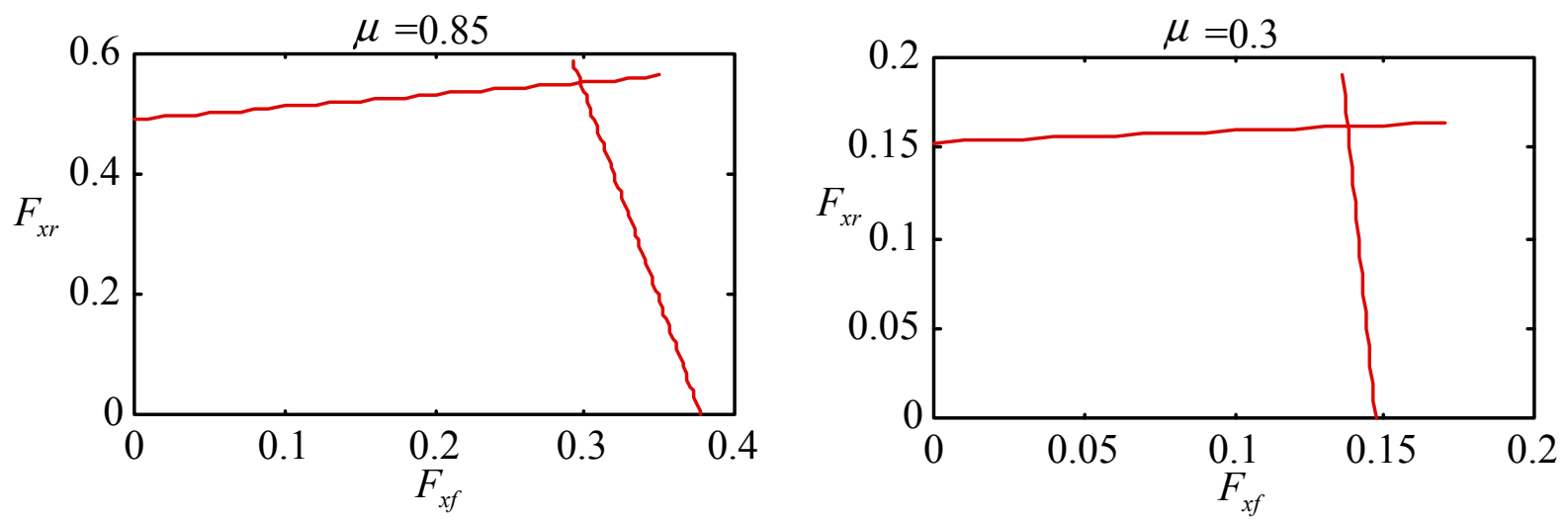
Figure 2: Achievable traction forces (a) $\mu=0.85$ and(b) $\mu=0.3$

The above analysis did not take into consideration the road gradient. If the design objective is to climb up a slippery slope, the load distributions should include the gradient angle, and a larger portion of the traction force should be distributed to the rear axle. The discussion is omitted since it is a straightforward extension of the above results.

The analysis for the braking case is similar to the procedures presented above. The braking force limit (without having wheel lock-up) are

$$
\begin{aligned}
& F_{x r} \leq \mu \frac{a m g-h F_{x f}}{a+b+\mu h} \\
& F_{x f} \leq \mu \frac{b m g+h F_{x r}}{a+b-\mu h}
\end{aligned}
$$

where positive $F_{x}{ }^{\prime} s$ represent braking. The optimal braking force distribution to achieve minimum stopping distance is

$$
\frac{F_{x f}}{F_{x r}}=\frac{b+\mu h}{a-\mu h}
$$

In other words, the front tires should bear more braking force on a dry road (69/31 (front/rear) when $\mu=0.85$ ). And the rear axle brakes become more important as the road becomes slippery (58/42 when $\mu=0.3$ ).

\section{Coordinated traction/braking control design}

In section 2, tire lateral forces are assumed to be small. When the vehicle is following a sharp curve, or swirling to avoid obstacles, large tire lateral forces are present. If large tire longitudinal forces are also requested (e.g. hard braking while following a curve), the longitudinal force distribution should take vehicle lateral forces into consideration. This kind of coordination is necessary because of the interaction between vehicle lateral and longitudinal dynamics. Two of the strongest interaction mechanisms are weight shift and the nonlinear tire force characteristics. When the vehicle is under lateral acceleration (e.g. following a curve), part of the load will shift from inner wheels to outer wheels. Similar weight shift occurs between front and rear axles when the vehicle is accelerating/decelerating. Therefore, force capacity of each wheel will be 
different. To achieve optimal performance, it is necessary to control each tire according to its capacity. This sounds unrealistic from today's practicality viewpoint. However, it is included in this study for two reasons. First, it represents the absolute optimum governed by physical limits, and can be used as the target reference for practical designs. Secondly, deep insight of the dynamic behaviors of the vehicle is learned by examining these upper limits.

\subsection{Basic equations}

Figures 3 and 4 show the tire longitudinal and lateral forces as functions of tire slip ratio and slip angle. In Figure 3, the tire slip angle $\alpha$ is 3 degrees. In Figure 4 , the tire slip ratio is 0.03 . Apparently, the tire slip angle and the tire slip ratio should be less than $\alpha_{p}$ and $\lambda_{p}$, respectively. When $\alpha>\alpha_{p}$ or $\lambda>\lambda_{p}$, both longitudinal and lateral forces obtained from the tire will be reduced.

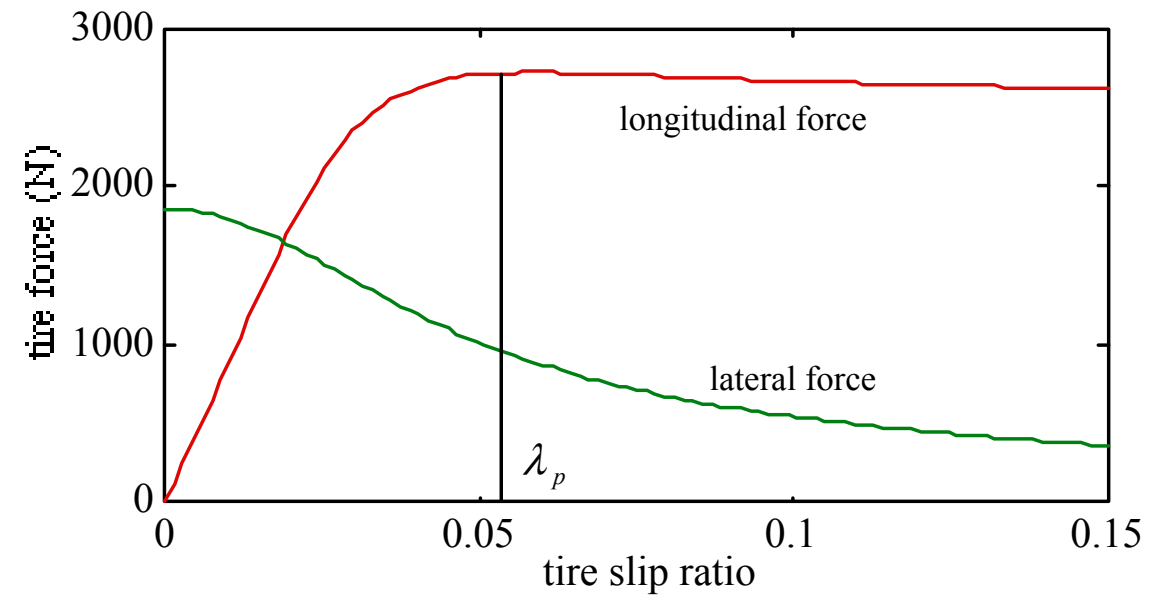

Figure 3 Tire force characteristics (slip angle $=3$ degrees) 


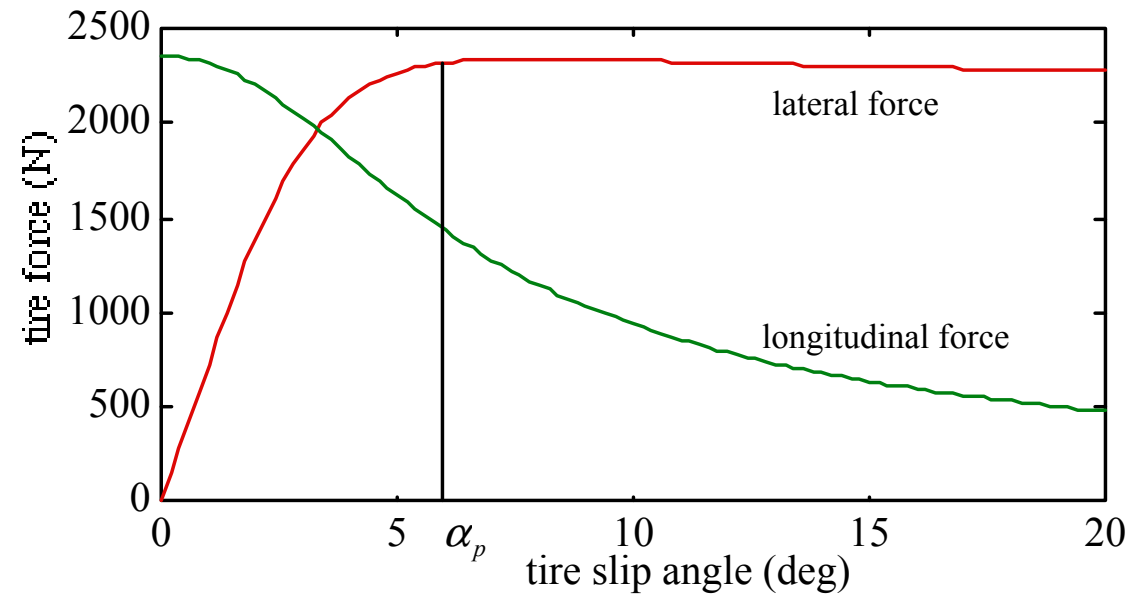

Figure 4 Tire force characteristics (slip ratio $=0.03$ )

When a vehicle is following a curve, tire lateral forces will have to generate the centrifugal force necessary to follow the curve. Weight-shift from inner wheels to outer wheels will occur to balance the roll moment. When the vehicle is accelerating, normal forces on the four tires can be obtained as follows:

$$
\begin{aligned}
& F_{z 1}=\frac{b m g}{2(a+b)}-\frac{\sum F_{x i} h}{2(a+b)}-\frac{k_{f} \sum F_{y i} h}{s_{b}} \\
& F_{z 2}=\frac{b m g}{2(a+b)}-\frac{\sum F_{x i} h}{2(a+b)}+\frac{k_{f} \sum F_{y i} h}{s_{b}} \\
& F_{z 3}=\frac{a m g}{2(a+b)}+\frac{\sum F_{x i} h}{2(a+b)}-\frac{k_{r} \sum F_{y i} h}{s_{b}} \\
& F_{z 4}=\frac{a m g}{2(a+b)}+\frac{\sum F_{x i} h}{2(a+b)}+\frac{k_{r} \sum F_{y i} h}{s_{b}}
\end{aligned}
$$

where $k_{f}$ is the vehicle lateral weight-shift distribution on front wheels, $k_{r}$ is the vehicle lateral weight-shift distribution on rear wheels. The tires are numbered as shown in Figure 5.

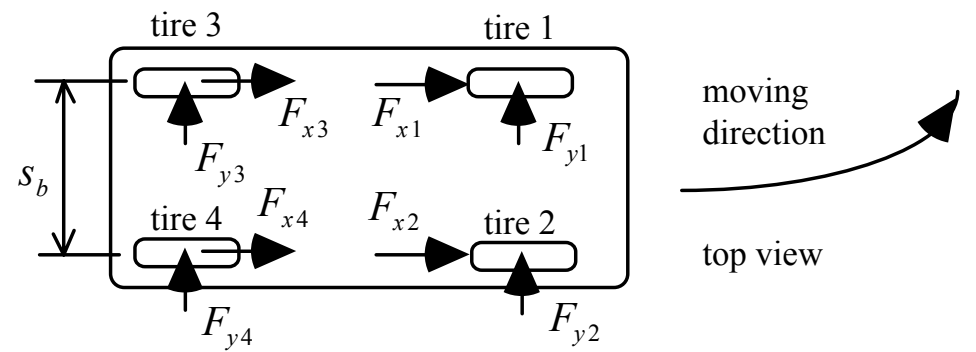

Figure 5: Schematic diagram of the four wheel vehicle model 
The normal forces for the braking case are:

$$
\begin{aligned}
& F_{z 1}=\frac{b m g}{2(a+b)}+\frac{\sum F_{x i} h}{2(a+b)}-\frac{k_{f} \sum F_{y i} h}{s_{b}} \\
& F_{z 2}=\frac{b m g}{2(a+b)}+\frac{\sum F_{x i} h}{2(a+b)}+\frac{k_{f} \sum F_{y i} h}{s_{b}} \\
& F_{z 3}=\frac{a m g}{2(a+b)}-\frac{\sum F_{x i} h}{2(a+b)}-\frac{k_{r} \sum F_{y i} h}{s_{b}} \\
& F_{z 4}=\frac{a m g}{2(a+b)}-\frac{\sum F_{x i} h}{2(a+b)}+\frac{k_{r} \sum F_{y i} h}{s_{b}}
\end{aligned}
$$

The tire forces are limited by the following equation:

$$
F_{x i}^{2}+F_{y i}^{2} \leq \mu^{2} F_{z i}^{2}, i=1 \ldots 4
$$

To follow the road curve, the tire lateral forces should satisfy:

$$
\sum F_{y i}=\frac{m V^{2}}{\rho}
$$

where $\rho$ is the road radius of curvature being followed, and $\mathrm{V}$ is the vehicle speed. To balance the vehicle yaw moment, the following equations need to be satisfied

During acceleration:

$$
\left(F_{y 1}+F_{y 2}\right) a-\left(F_{y 3}+F_{y 4}\right) b+\left(F_{x 2}+F_{x 4}\right) \frac{S_{b}}{2}-\left(F_{x 1}+F_{x 3}\right) \frac{S_{b}}{2}=0
$$

During braking:

$$
\left(F_{y 1}+F_{y 2}\right) a-\left(F_{y 3}+F_{y 4}\right) b-\left(F_{x 2}+F_{x 4}\right) \frac{s_{b}}{2}+\left(F_{x 1}+F_{x 3}\right) \frac{s_{b}}{2}=0
$$

\subsection{Single-wheel-steering, single-wheel-drive (SWS/SWD)Vehicles}

When the steering angle and traction torque of all four wheels are individually controlled, the optimization problem can be stated as follows:

Case 1: (SWS/SWD vehicles during acceleration)

$$
\text { Maximize }\left(F_{x 1}+F_{x 2}+F_{x 3}+F_{x 4}\right)
$$

Under the constraints:

Eqs. (11) through (14);

Eqs. (15), (16), and (17). 
Case 2: (SWS/SWD vehicles during deceleration)

$$
\operatorname{Maximize}\left(F_{x 1}+F_{x 2}+F_{x 3}+F_{x 4}\right)
$$

Under the constraints:

Eqs. (11a) through (14a);

Eqs. (15), (16), and (17a).

\subsection{Four-wheel-steering, Single-wheel-drive (4WS/SWD)Vehicles}

When the wheels of the vehicle are steered in pairs (front and rear), the slip angles of the tires on the same axle are about the same. By using the friction ellipse approximation:

$$
F_{y}=F_{y 0}\left[1-\left(\frac{F_{x}}{F_{x \max }}\right)^{2}\right]^{0.5}
$$

the following relationship can be obtained:

$$
\begin{aligned}
& \frac{F_{y 1}}{F_{y 2}} \approx \frac{\sqrt{\mu^{2} F_{z 1}^{2}-F_{x 1}^{2}}}{\sqrt{\mu^{2} F_{z 2}^{2}-F_{x 2}^{2}}} \\
& \frac{F_{y 3}}{F_{y 4}} \approx \frac{\sqrt{\mu^{2} F_{z 3}^{2}-F_{x 3}^{2}}}{\sqrt{\mu^{2} F_{z 4}^{2}-F_{x 4}^{2}}}
\end{aligned}
$$

where $F_{y 0}$ is the lateral force corresponding to pure lateral slip case. Eqs.(18) and (19) are obtained based on the assumption that the normalized tire lateral force is (almost) linearly proportional to the tire slip angle before the peak (see Figure 6). The driving torque of the four wheels are assumed to be controlled independently. IC vehicles with three differentials and electric vehicles equipped with driving motors for each wheel fall into this category. The corresponding optimization problems are: 


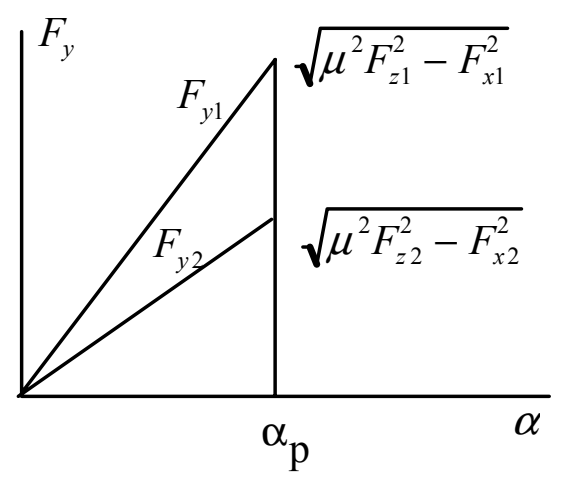

Figure 6 Linear tire lateral force characteristic

Case 3: (4WS/SWD vehicles during acceleration)

$$
\operatorname{Maximize}\left(F_{x 1}+F_{x 2}+F_{x 3}+F_{x 4}\right)
$$

Under the constraints:

Eqs. (11) through (14);

$$
\begin{aligned}
& F_{y 1}=\frac{\left(2 b m V^{2}+\left(F_{x 1}+F_{x 3}-F_{x 2}-F_{x 4}\right) \rho s_{b}\right)}{2 \rho(a+b)} \frac{\sqrt{\mu^{2} F_{z 1}^{2}-F_{x 1}^{2}}}{\sqrt{\mu^{2} F_{z 1}^{2}-F_{x 1}^{2}}+\sqrt{\mu^{2} F_{z 2}^{2}-F_{x 2}^{2}}} \\
& F_{y 2}=\frac{\left(2 b m V^{2}+\left(F_{x 1}+F_{x 3}-F_{x 2}-F_{x 4}\right) \rho s_{b}\right)}{2 \rho(a+b)} \frac{\sqrt{\mu^{2} F_{z 2}^{2}-F_{x 2}^{2}}}{\sqrt{\mu^{2} F_{z 1}^{2}-F_{x 1}^{2}}+\sqrt{\mu^{2} F_{z 2}^{2}-F_{x 2}^{2}}} \\
& F_{y 3}=\frac{\left(2 a m V^{2}+\left(F_{x 2}+F_{x 4}-F_{x 1}-F_{x 3}\right) \rho s_{b}\right)}{2 \rho(a+b)} \frac{\sqrt{\mu^{2} F_{z 3}^{2}-F_{x 3}^{2}}}{\sqrt{\mu^{2} F_{z 3}^{2}-F_{x 3}^{2}}+\sqrt{\mu^{2} F_{z 4}^{2}-F_{x 4}^{2}}} \\
& F_{y 4}=\frac{\left(2 a m V^{2}+\left(F_{x 2}+F_{x 4}-F_{x 1}-F_{x 3}\right) \rho s_{b}\right)}{2 \rho(a+b)} \frac{\sqrt{\mu^{2} F_{z 4}^{2}-F_{x 4}^{2}}}{\sqrt{\mu^{2} F_{z 3}^{2}-F_{x 3}^{2}}+\sqrt{\mu^{2} F_{z 4}^{2}-F_{x 4}^{2}}}
\end{aligned}
$$

Eq. (15).

Case 4: (4WS/SWD vehicles during deceleration)

$$
\operatorname{Maximize}\left(F_{x 1}+F_{x 2}+F_{x 3}+F_{x 4}\right)
$$

Under the constraints:

Eqs. (11a) through (14a);

$$
\begin{aligned}
& F_{y 1}=\frac{\left(2 b m V^{2}-\left(F_{x l}+F_{x 3}-F_{x 2}-F_{x 4}\right) \rho s_{b}\right)}{2 \rho(a+b)} \frac{\sqrt{\mu^{2} F_{z 1}^{2}-F_{x 1}^{2}}}{\sqrt{\mu^{2} F_{z 1}^{2}-F_{x 1}^{2}}+\sqrt{\mu^{2} F_{z 2}^{2}-F_{x 2}^{2}}} \\
& F_{y 2}=\frac{\left(2 b m V^{2}-\left(F_{x 1}+F_{x 3}-F_{x 2}-F_{x 4}\right) \rho s_{b}\right)}{2 \rho(a+b)} \frac{\sqrt{\mu^{2} F_{z 2}^{2}-F_{x 2}^{2}}}{\sqrt{\mu^{2} F_{z 1}^{2}-F_{x 1}^{2}}+\sqrt{\mu^{2} F_{z 2}^{2}-F_{x 2}^{2}}}
\end{aligned}
$$




$$
\begin{aligned}
& F_{y 3}=\frac{\left(2 a m V^{2}-\left(F_{x 2}+F_{x 4}-F_{x 1}-F_{x 3}\right) \rho s_{b}\right)}{2 \rho(a+b)} \frac{\sqrt{\mu^{2} F_{z 3}^{2}-F_{x 3}^{2}}}{\sqrt{\mu^{2} F_{z 3}^{2}-F_{x 3}^{2}}+\sqrt{\mu^{2} F_{z 4}^{2}-F_{x 4}^{2}}} \\
& F_{y 4}=\frac{\left(2 a m V^{2}-\left(F_{x 2}+F_{x 4}-F_{x 1}-F_{x 3}\right) \rho s_{b}\right)}{2 \rho(a+b)} \frac{\sqrt{\mu^{2} F_{z 4}^{2}-F_{x 4}^{2}}}{\sqrt{\mu^{2} F_{z 3}^{2}-F_{x 3}^{2}}+\sqrt{\mu^{2} F_{z 4}^{2}-F_{x 4}^{2}}}
\end{aligned}
$$

Eq. (15).

\subsection{Four-wheel-steering, Four-wheel-drive (4WS/4WD)Vehicles}

Most of the existing four-wheel-drive mechanisms split traction torque between front/rear axles. This ratio is usually fixed (say, 50/50), but it could vary according to road surface conditions for improved performance. One such electronically controlled torque split 4WD system has been reported in (Yaguchi et al. 1989). Conventional differentials split the driving torque to the wheels on the two sides evenly. When vehicles are equipped with limited slip differentials or traction control systems, however, the wheel torque on the same axle are not readily determined. Suppose no brake torque is applied and the uncoordinated optimal torque split ratio presented in Eq.(7) is used, the following constraint needs to be included in the acceleration case:

$$
\left(F_{x 1}+F_{x 2}\right) \frac{a+\mu h}{b-\mu h}=F_{x 3}+F_{x 4}
$$

It should be noted that we did not impose any constraint on the torque split ratio between right/left wheels to represent the most general case. In other words, the 4WD configuration used in this section is in its widest sense. Under the extra constraint shown in Eq.(20), the optimization problem is formulated in the following:

Case 5: (4WS/4WD vehicles during acceleration)

$$
\operatorname{Maximize}\left(F_{x 1}+F_{x 2}+F_{x 3}+F_{x 4}\right)
$$

Under the constraints:

Eqs. (11) through (14);

$$
F_{y 1}=\frac{\left(2 b m V^{2}+\left(F_{x l}+F_{x 3}-F_{x 2}-F_{x 4}\right) \rho s_{b}\right)}{2 \rho(a+b)} \frac{\sqrt{\mu^{2} F_{z 1}^{2}-F_{x 1}^{2}}}{\sqrt{\mu^{2} F_{z 1}^{2}-F_{x 1}^{2}}+\sqrt{\mu^{2} F_{z 2}^{2}-F_{x 2}^{2}}}
$$




$$
\begin{aligned}
& F_{y 2}=\frac{\left(2 b m V^{2}+\left(F_{x 1}+F_{x 3}-F_{x 2}-F_{x 4}\right) \rho s_{b}\right)}{2 \rho(a+b)} \frac{\sqrt{\mu^{2} F_{z 2}^{2}-F_{x 2}^{2}}}{\sqrt{\mu^{2} F_{z 1}^{2}-F_{x 1}^{2}}+\sqrt{\mu^{2} F_{z 2}^{2}-F_{x 2}^{2}}} \\
& F_{y 3}=\frac{\left(2 a m V^{2}+\left(F_{x 2}+F_{x 4}-F_{x 1}-F_{x 3}\right) \rho s_{b}\right)}{2 \rho(a+b)} \frac{\sqrt{\mu^{2} F_{z 3}^{2}-F_{x 3}^{2}}}{\sqrt{\mu^{2} F_{z 3}^{2}-F_{x 3}^{2}}+\sqrt{\mu^{2} F_{z 4}^{2}-F_{x 4}^{2}}} \\
& F_{y 4}=\frac{\left(2 a m V^{2}+\left(F_{x 2}+F_{x 4}-F_{x 1}-F_{x 3}\right) \rho s_{b}\right)}{2 \rho(a+b)} \frac{\sqrt{\mu^{2} F_{z 4}^{2}-F_{x 4}^{2}}}{\sqrt{\mu^{2} F_{z 3}^{2}-F_{x 3}^{2}}+\sqrt{\mu^{2} F_{z 4}^{2}-F_{x 4}^{2}}}
\end{aligned}
$$

Eqs. (15) and (20)

The brake mechanism, which is not restricted to split torque between front and rear axles, will have the same problem formulation as case 4 . Therefore, it is not repeated.

\subsection{Human-Steering, Four-Wheel-Drive (Human/4WD)Vehicles}

An interesting special case can be formulated by substituting Eqs. (17) or (17a) with the following three equations:

$$
\begin{aligned}
& F_{y 1}+F_{y 2}=\frac{m V^{2}}{\rho} \frac{b}{a+b} \\
& F_{y 3}+F_{y 4}=\frac{m V^{2}}{\rho} \frac{a}{a+b} \\
& F_{x 1}+F_{x 3}=F_{x 2}+F_{x 4}
\end{aligned}
$$

In other words, the yaw moments generated by lateral forces and longitudinal forces are balanced separately. This special case is of interest because the extra constraints imply that the traction/braking will not affect the steering algorithm. This is important when the traction/braking is controlled by control systems, while the steering is controlled by a human driver. The results from this optimization can be interpreted as the maximum longitudinal force that could be requested from the traction/braking control systems of human steered vehicles. Furthermore, the effects of tire longitudinal forces on lateral dynamics have been assumed to be small in the development of many automatic steering control algorithms. Results from this case can also be used as the upper limit for such kind of independent lateral/longitudinal control systems. Again, the 4WD 
configuration is used in its most general sense. For vehicles equipped with conventional differentials, we could use

$$
\begin{aligned}
& F_{x 1}=F_{x 2} \\
& F_{x 3}=F_{x 4}
\end{aligned}
$$

instead of Eq.(23) to get a more restricted optimization problem. The optimization problems now become:

Case 6: (Human steering/4WD vehicles during acceleration)

$$
\text { Maximize }\left(F_{x 1}+F_{x 2}+F_{x 3}+F_{x 4}\right)
$$

Under the constraints:

Eqs. (11) through (14);

$$
\begin{aligned}
& F_{y 1}=\frac{m V^{2}}{\rho} \frac{b}{a+b} \frac{\sqrt{\mu^{2} F_{z 1}^{2}-F_{x 1}^{2}}}{\sqrt{\mu^{2} F_{z 1}^{2}-F_{x 1}^{2}}+\sqrt{\mu^{2} F_{z 2}^{2}-F_{x 2}^{2}}} ; \\
& F_{y 2}=\frac{m V^{2}}{\rho} \frac{b}{a+b} \frac{\sqrt{\mu^{2} F_{z 2}^{2}-F_{x 2}^{2}}}{\sqrt{\mu^{2} F_{z 1}^{2}-F_{x 1}^{2}}+\sqrt{\mu^{2} F_{z 2}^{2}-F_{x 2}^{2}}} ; \\
& F_{y 3}=\frac{m V^{2}}{\rho} \frac{a}{a+b} \frac{\sqrt{\mu^{2} F_{z 3}^{2}-F_{x 3}^{2}}}{\sqrt{\mu^{2} F_{z 3}^{2}-F_{x 3}^{2}}+\sqrt{\mu^{2} F_{z 4}^{2}-F_{x 4}^{2}}} ; \\
& F_{y 4}=\frac{m V^{2}}{\rho} \frac{a}{a+b} \frac{\sqrt{\mu^{2} F_{z 4}^{2}-F_{x 4}^{2}}}{\sqrt{\mu^{2} F_{z 3}^{2}-F_{x 3}^{2}}+\sqrt{\mu^{2} F_{z 4}^{2}-F_{x 4}^{2}}} ;
\end{aligned}
$$

Eqs. (15), (20) and (23).

Case 7: (Human steering/4WD vehicles during deceleration)

$$
\operatorname{Maximize}\left(F_{x 1}+F_{x 2}+F_{x 3}+F_{x 4}\right)
$$

Under the constraints:

Eqs. (11a) through (14a);

$$
\begin{aligned}
& F_{y 1}=\frac{m V^{2}}{\rho} \frac{b}{a+b} \frac{\sqrt{\mu^{2} F_{z 1}^{2}-F_{x 1}^{2}}}{\sqrt{\mu^{2} F_{z 1}^{2}-F_{x 1}^{2}}+\sqrt{\mu^{2} F_{z 2}^{2}-F_{x 2}^{2}}} ; \\
& F_{y 2}=\frac{m V^{2}}{\rho} \frac{b}{a+b} \frac{\sqrt{\mu^{2} F_{z 2}^{2}-F_{x 2}^{2}}}{\sqrt{\mu^{2} F_{z 1}^{2}-F_{x 1}^{2}}+\sqrt{\mu^{2} F_{z 2}^{2}-F_{x 2}^{2}}} ; \\
& F_{y 3}=\frac{m V^{2}}{\rho} \frac{a}{a+b} \frac{\sqrt{\mu^{2} F_{z 3}^{2}-F_{x 3}^{2}}}{\sqrt{\mu^{2} F_{z 3}^{2}-F_{x 3}^{2}}+\sqrt{\mu^{2} F_{z 4}^{2}-F_{x 4}^{2}}} ;
\end{aligned}
$$




$$
F_{y 4}=\frac{m V^{2}}{\rho} \frac{a}{a+b} \frac{\sqrt{\mu^{2} F_{z 4}^{2}-F_{x 4}^{2}}}{\sqrt{\mu^{2} F_{z 3}^{2}-F_{x 3}^{2}}+\sqrt{\mu^{2} F_{z 4}^{2}-F_{x 4}^{2}}} ;
$$

Eqs. (15) and (23).

Case 8: (Human steering/4WD vehicles with conventional differentials during acceleration)

Exactly the same as case 6, but use Eqs.(24) and (25) instead of (23).

\section{Results and Discussions}

In the simulations, the vehicle is assumed to be following a curve corresponding to $3 \mathrm{~m} / \mathrm{sec}^{2}$ in lateral acceleration. Figure 7 shows the results for case 1. It can be seen that the tire force ratios are uniform for lateral, longitudinal, and normal forces. In other words, $F_{x i} / F_{x j}=F_{y i} / F_{y j}=F_{z i} / F_{z j}$ for all $i$ and $j(i \neq j)$. The same phenomenon is also observed in the braking case (Case 2), the results of which are shown in Fig. 8. The front wheels should account for more traction forces and less braking forces on slippery road (small $\mu$ ) than on dry road (large $\mu$ ). These trends are the same for both inner and outer wheels and coincide with the observation for the straight road case presented in section 2. The left/right ratios indicate different characteristics for front wheels and rear wheels. For example, in traction control, more tire forces are shifted toward the outer-front and inner-rear wheels when 

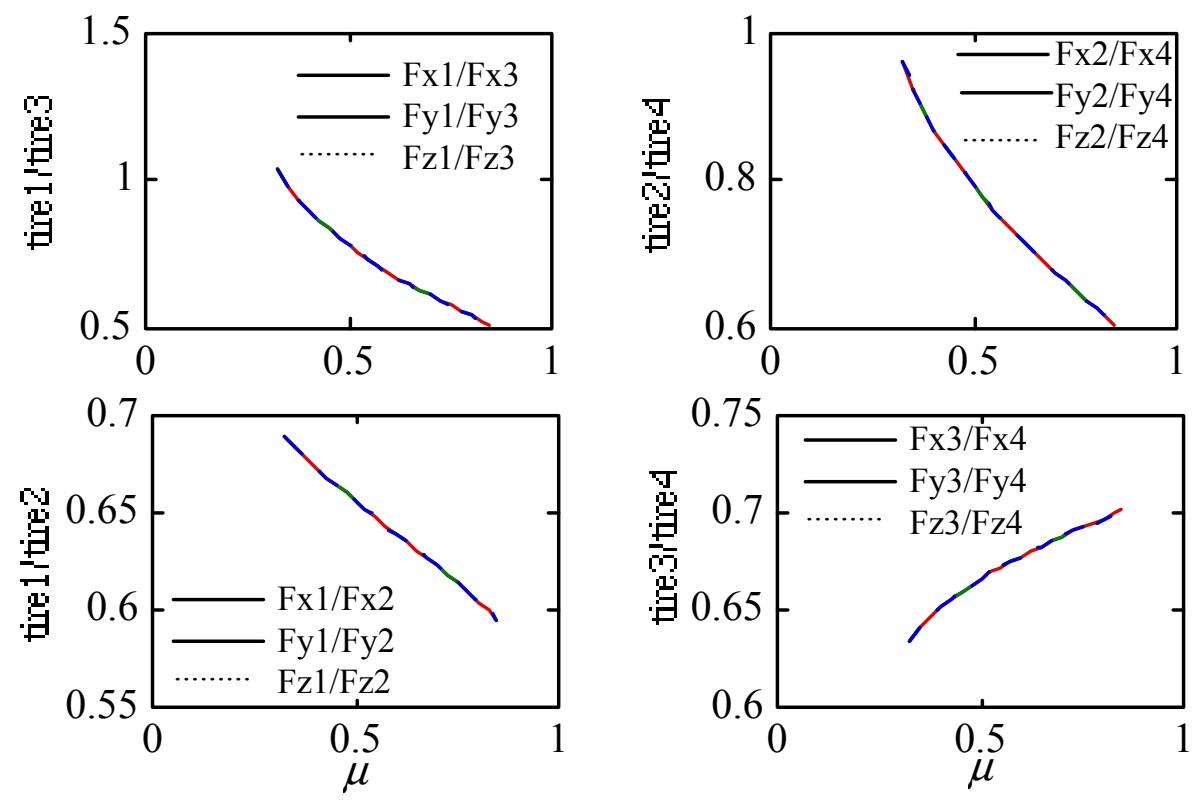

Figure 7 Tire force distribution for Case 1 (SWS/SWD, traction)
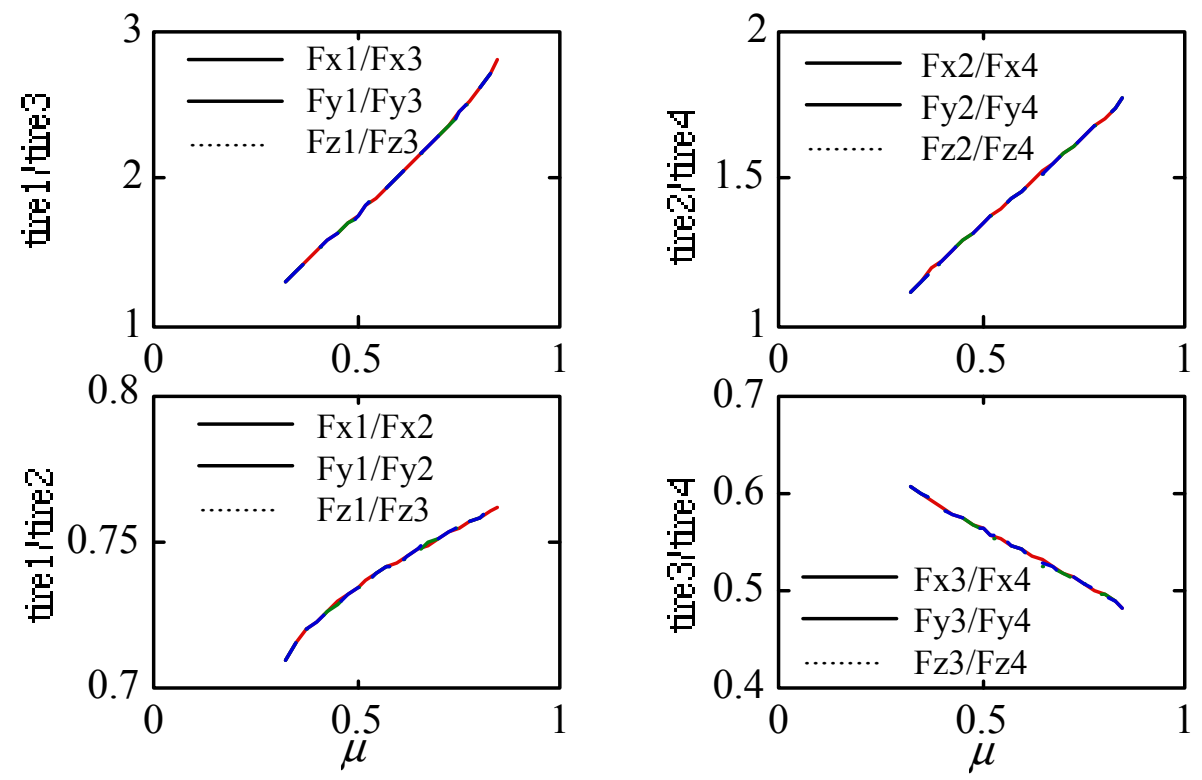

Figure 8 Tire force distribution for Case 2 (SWS/SWD, braking)

friction increases. The fact that all force ratios between tires fall into the same curve indicates that the capacities of all the tires are used completely. In other words, because of the large number of manipulated variables (four steering 
angles, four traction/braking forces), we are able to achieve the absolute maximum acceleration/deceleration.

When we have only two steering (front and rear) and four driving/braking control inputs (Cases 3 and 4), we achieve the same level of acceleration/deceleration performance. Actually, the force distributions are exactly the same as Cases 1 and 2. In other words, the freedom of being able to steer all the four tires individually does not improve vehicle traction/braking performance. The six free variables are enough to achieve both curve following and maximum acceleration/deceleration simultaneously.

When the front-rear traction torque is split according to a fixed ratio (Case 5), the absolute optimal performance can no longer be achieved. Figure 9 shows the results, where all the tire forces are normalized against the optimal forces obtained in Case 1. The following observations can be made: (1) Less acceleration is achieved (less weight shift from front to rear); (2) Much of the capacity of tire 2 (front outer wheel) is wasted; (3) The capacity of the rear wheels (wheels 3 and 4) is much more thoroughly used. The reduced performance is due
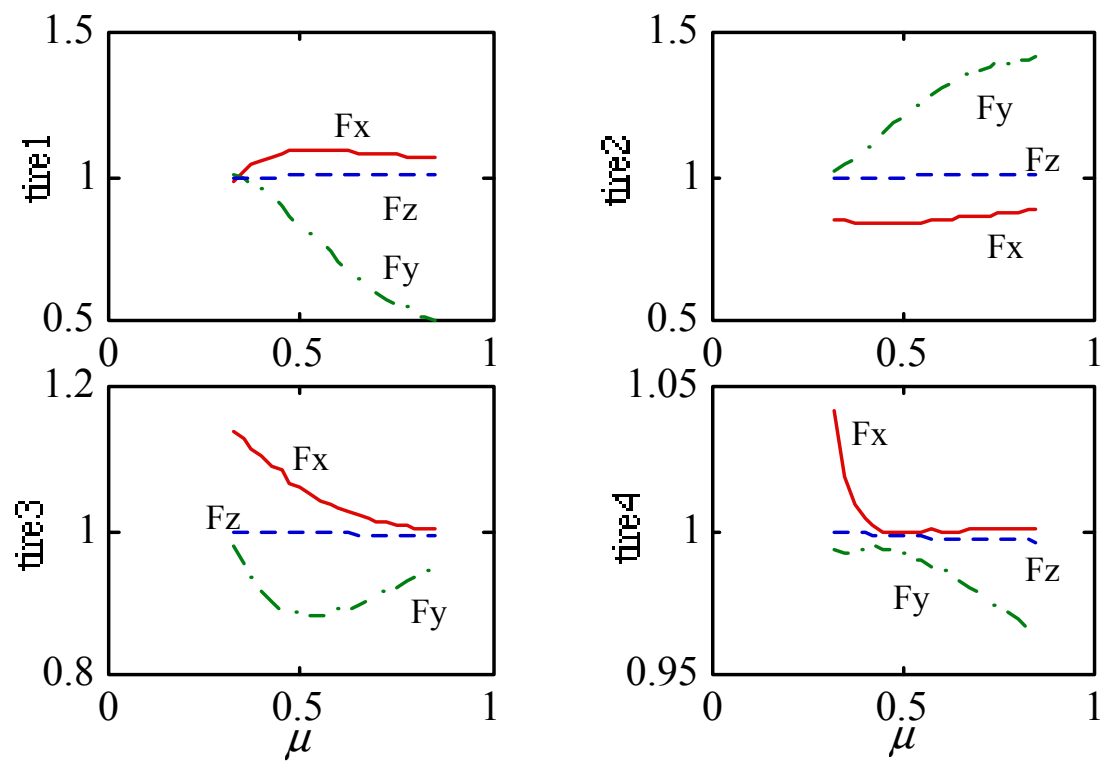

Figure 9 Tire force distribution for Case 5 (4WS/4WD, traction) 
to the fact that traction torque distribution (Eq.(7)) does not consider lateral acceleration. When the vehicle is following a curve, less longitudinal force can be expected from the tires. Eq.(7) predicts a higher acceleration, and the torque distributed to the front axle is less than the capacity of the front tires. The optimization scheme reduces this mismatch by requesting more lateral force from tire 2, and less from the rear wheels. However, due to the 4WS configuration, part of the capacity of tire 2 is still wasted. It should be noted that the torque split ratio governed by the $4 \mathrm{WD}$ mechanism can be changed by applying brake over increased engine torque. This inefficient mode of operation is not discussed in this paper.

Simulations for the case when the 4WD mechanism splits the torque according to a fixed ratio (say, 50/50) have also been performed. It was found that the performance deteriorates significantly (up to 20\%). This is due to the fact that the fixed split ratio is optimal only for a certain road friction coefficient. When $\mu$ varies, much of the tire force capacity in one of the axle is wasted.

When the yawing moments of the lateral and longitudinal forces are required to balance separately, significant force redistribution is expected, since a large moment is generated by the longitudinal forces in both Cases 1 and 2 (see Figure 10). Positive (counterclockwise) yaw moment of Case 1 implies that in Case 6, (1) larger lateral force has to be obtained from the front tires; (2) longitudinal forces at the outer tires need to be reduced compared with inner tires. Figure 11 shows that these observations indeed occur. Since the outer tires bear more force capacity due to weight shift, we have to trade longitudinal acceleration for lateral stability in the human controlled cases. 

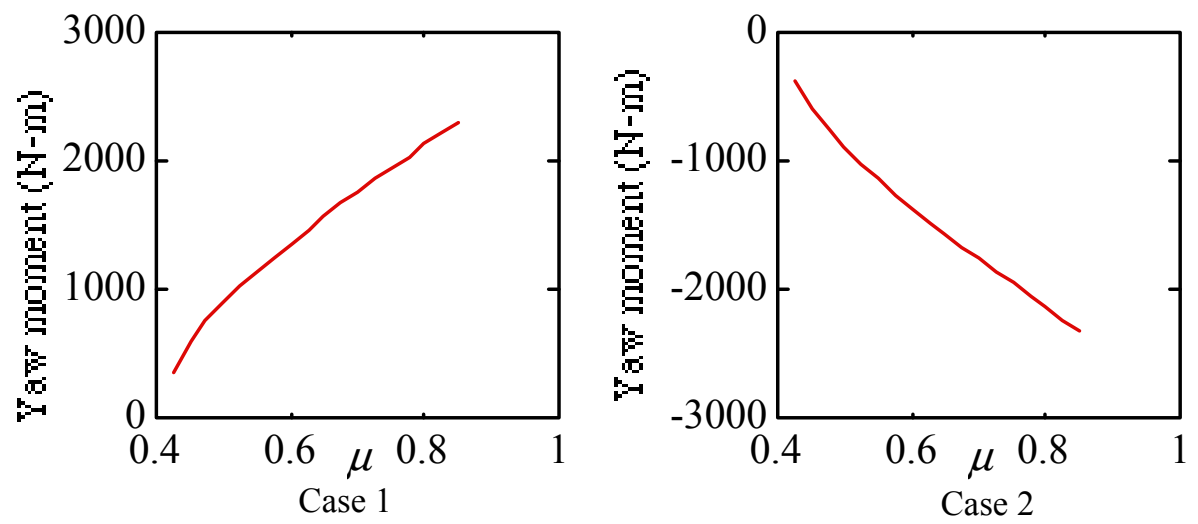

Figure 10 Yaw moment due to the longitudinal forces (Cases 1 and 2)
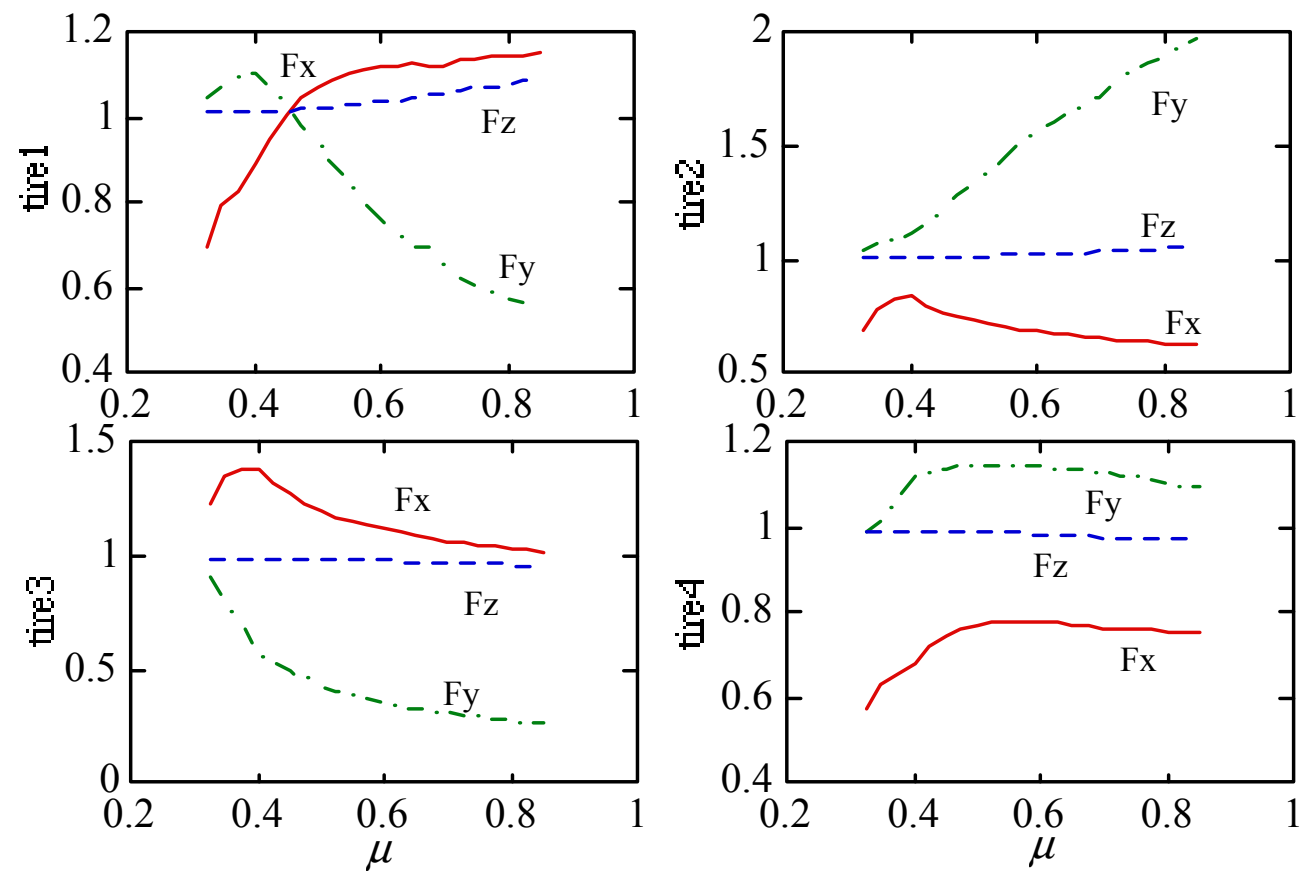

Figure 11 Tire force distribution for Case 6 (human steering, traction)

Figure 12 shows the results of Case 7 (human steered vehicle, braking). Similar to the reasoning for Case 6, we notice that (1) more lateral forces need to come from the rear tires; (2) less longitudinal forces should be requested from the outer tires. 

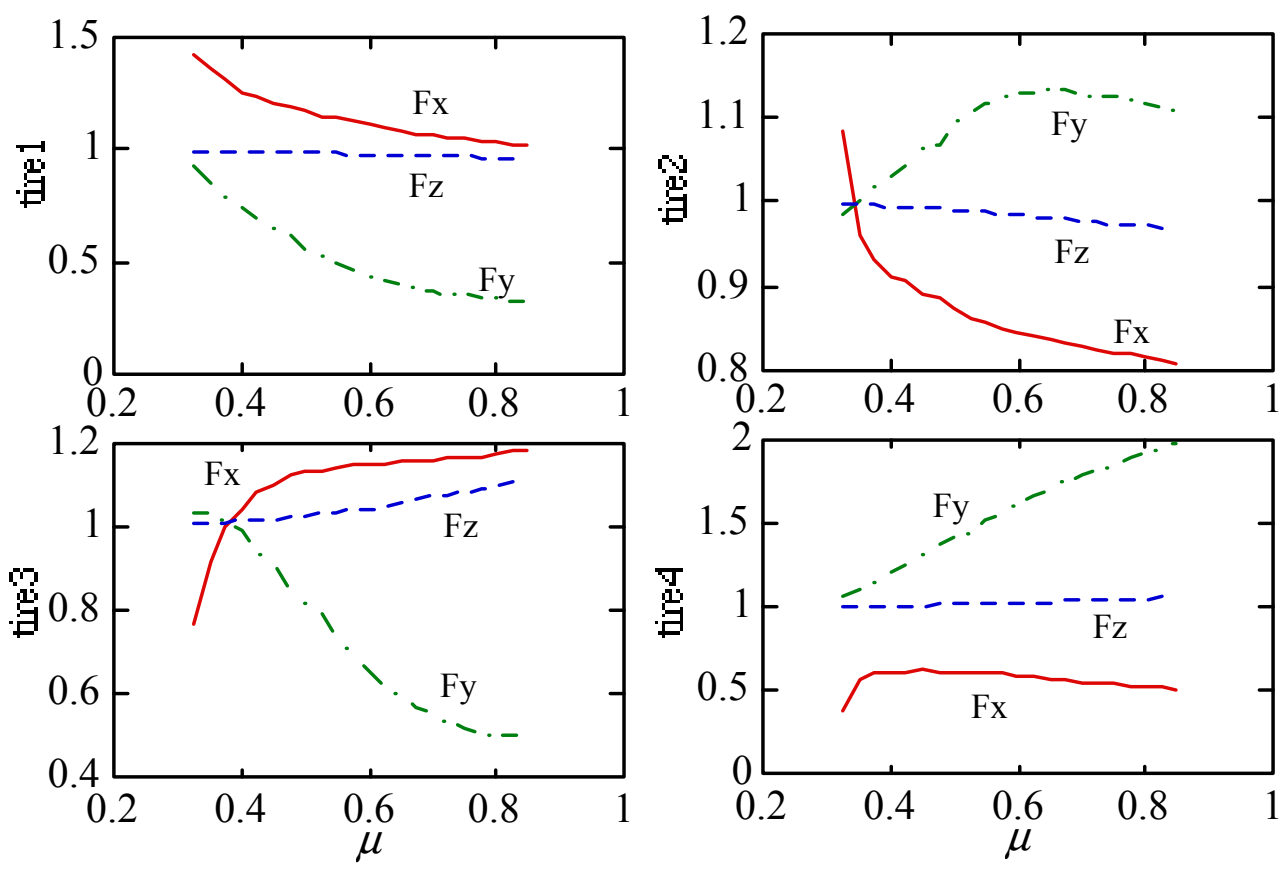

Figure 12 Tire force distribution for Case 7 (human steering, braking)

Case 8 can be viewed as a special case of case 6 . More specifically, the torque on the wheels of the same axle (tire 21 and 2, or tires 3 and 4) are forced to be the same. This case represents the vehicles equipped with conventional differentials. It can be seen from Figure 13 that the force distributions are very similar to those of case 6 . The difference is slightly larger in the high- $\mu$ region than in the low- $\mu$ region. In other words, the performance limitation imposed by conventional differentials is larger on high- $\mu$ road surfaces.

Figure 14 shows vehicle acceleration of all the seven cases. It can be seen that (1) single wheel steering provides no benefits in terms of longitudinal acceleration under curve following; (2) when we split traction torque between front/rear axles according to the road friction coefficient, very minimal performance deterioration ( $5 \%$ for $0.4 \mathrm{G}, 1 \%$ for $0.3 \mathrm{G}$ lateral acceleration) is expected, assuming that the tire steering is controlled in a cooperative manner; (3) significant performance loss $(27 \%$ for $0.4 \mathrm{G}, 16 \%$ for $0.3 \mathrm{G}$ lateral acceleration) will be experienced if the traction/braking system is constrained not to generate 
any yaw moment; and (4) Conventional differentials do not impose significant acceleration performance constraint.

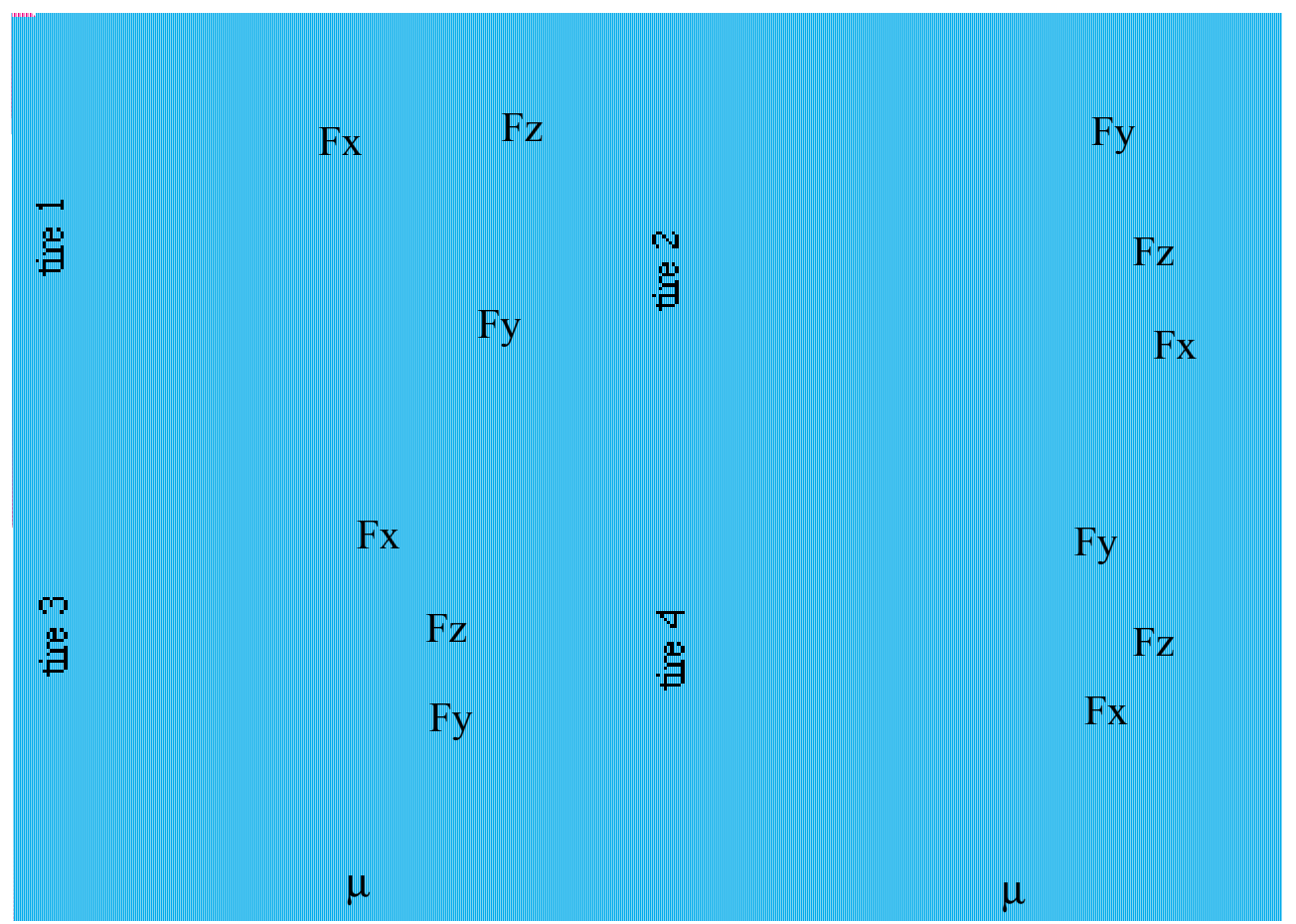

Figure 13 Tire force distribution for Case 8 (human steering, traction, conv. differentials)
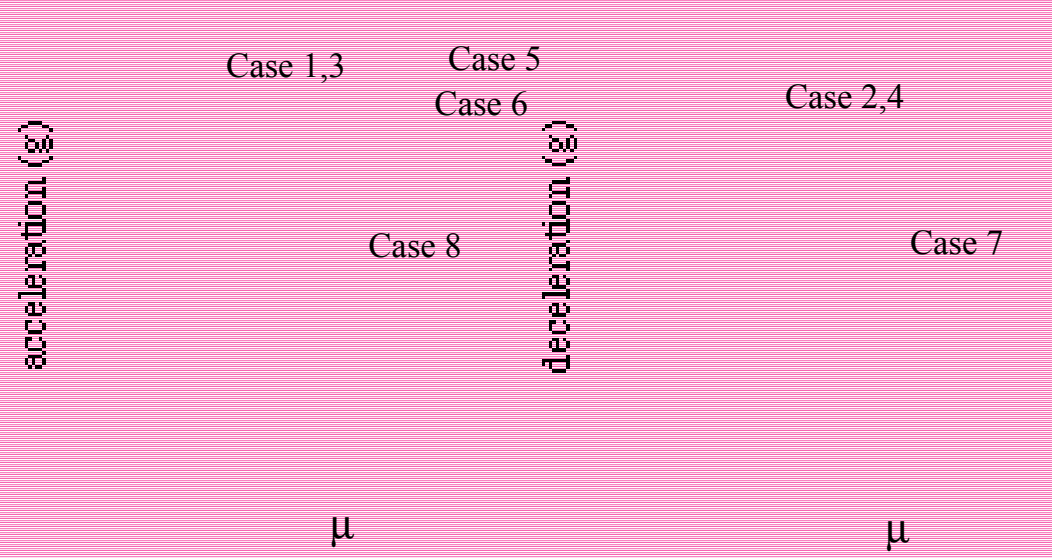

Figure 14 Vehicle acceleration/deceleration for all cases 
All the results presented up to now have been reported with fixed lateral acceleration and varying road friction coefficient. Figure 15 shows the results of Cases 6 and 7 (human steering, traction and braking) for varying vehicle speed. The road radius of curvature is assumed to be 400 meters, and $\mu$ is assumed to be 0.5. When the vehicle speed is low, the problem reduces to the decoupled case (see section 2). As vehicle speed (equivalently, lateral acceleration) increases, significant torque redistribution among tires becomes necessary for optimal performance.
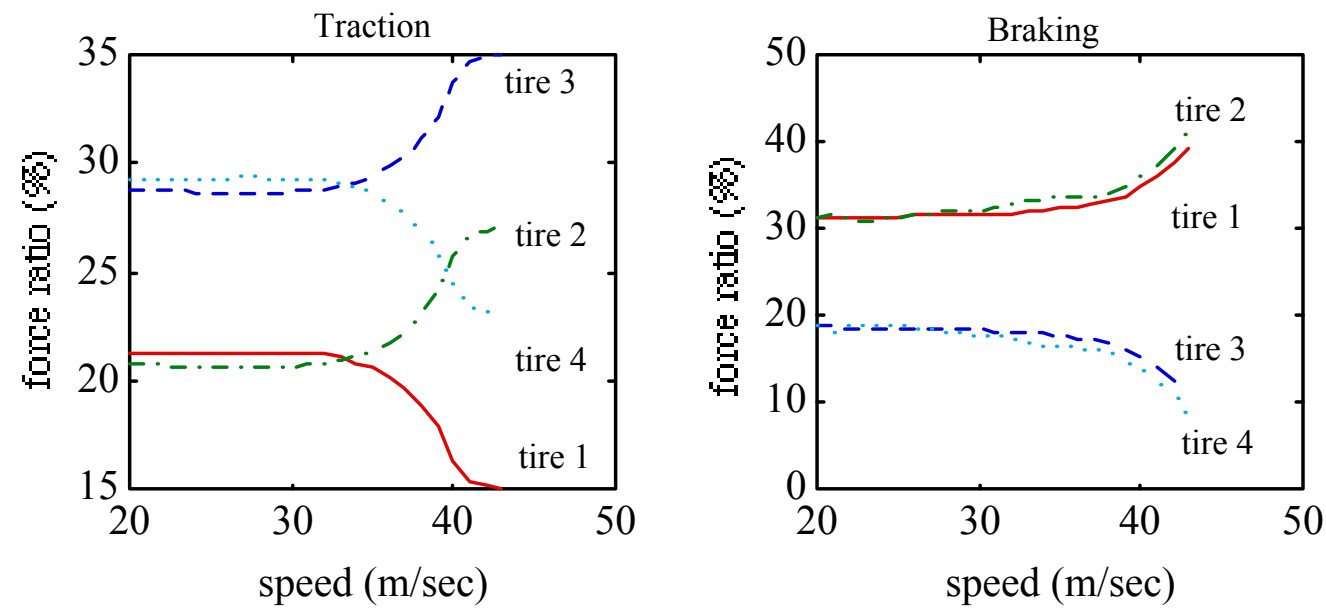

Figure 15 Tire force distributions with varying speed

\section{Conclusions}

In this paper, optimal tire force distribution for a vehicle under steadystate cornering is discussed. The objective is to achieve maximum longitudinal acceleration without compromising the curve following ability of the vehicle. Four vehicle configurations are considered: single-wheel-steering, single-wheeldrive; four-wheel-steering, single-wheel-drive; four-wheel-steering, four-wheeldrive; and human steering, four-wheel-drive. It was found that single-wheelsteering offers no benefits over four-wheel-steering vehicles. Torque split fourwheel-drive mechanism only reduces the performance by about $1 \%$ (when the lateral acceleration is $0.3 \mathrm{G}$ ) if the split ratio is adjusted according to the road friction. When the split ratio is fixed (say, 50/50), the performance may deteriorate by up to $20 \%$. When the steering is controlled by human drivers and the longitudinal forces are constrained not to generate yaw moment, the 
maximum acceleration and deceleration are about $16 \%$ lower than the optimal cases (with adaptive torque split ratio, $0.3 \mathrm{G}$ lateral acceleration). Under the same (no yaw moment) constraint, the extra performance limit introduced by conventional differentials is very minimal.

\section{References}

Anto, S., "The Next Step in Automotive Electronic Control", International Congress on Transportation Electronics, Dearborn, 1988.

Eguchi, T. et al., "Development of Super Hicas, a New Rear Wheel Steering System with Phase reversal Control," SAE paper 891987, 1989.

Kimbrough, S., "A Brake Control Strategy for Emergency Stops That Involve Steering, Part 1: Theory, Part 2: Implementation Issues and Simulation Results," ASME Winter Annual Meeting, AMD Vol.108, 1990.

Kizu, R., Harada, H., and Minabe, H., "Electronic Control of Car Chassis: Present Status and Future Perspective", International Congress on Transportation Electronics, Dearborn.

Matsumoto, S., Yamaguchi, H., Inoue, H., and Yasuno, Y., "Braking Force Distribution Control for Improved Vehicle Dynamics," Proceedings of the International Symposium on Advanced Vehicle Control, 1992, SAE paper 923079.

Motoyama, S. et al., "Effect of Traction Force Distribution Control on Vehicle Dynamics," Proceedings of the International Symposium on Advanced Vehicle Control, 1992, pp.447-451, SAE paper \#923080.

Nakazato, H., Iwata K., and Yuichi, Y., "A New System for Independently Controlling Braking Force Between Inner and Outer Wheels", SAE paper 890835, 1989.

Peterson, E., Reinecke, E., Liermann, P., "Anti-Lock Braking System (ABS) with Integrated Drive Slip Control (ASR) for Commercial Vehicles," Electronics in Commercial Vehicle Braking and Steering Systems, SAE SP-753, 1988.

Ressler, N. W., Patterson D. J., and Soltis, M. W., "Integrated Chassis and Suspension Controls - Present and Future World of Chassis Electronic Controls", International Congress on Transportation Electronics, Dearborn, 1988. 
Rittmannsberger, N., "Antilock Braking System and Traction Control," International Congress on Transportation Electronics Proceedings, Oct. 17-18, Dearborn, MI, 1988.

Sano, S., Furukawa, Y., Shiraishi, S., "Four Wheel Steering System with Rear Wheel Steer Angle Controlled as a Function of Steering Wheel Angle," SAE paper 860625, 1986.

Schilke, N.A., Fruechte, R.D., Boustany, N.M., Karmel, A.M., Repa, B.S., and Rillings, J.H., "Integrated Vehicle Control", International Congress on Transportation Electronics, Dearborn, 1988.

Shiraishi, S. et al., "Control Concept for Traction Control System (TCS) and Its Performance," 12th International Technical Conference on Experimental Safety Vehicles, Sweden, 1989.

Takiguchi, T. et al., "Improvement of Vehicle Dynamics by Vehicle-SpeedSensing Four-Wheel Steering System," SAE paper 860624, 1986.

Yamamoto, M., "Active Control Strategy for Improved Handling and Stability", SAE paper 911902, 1991.

Yaguchi, E. et al., "Active Safety Through Traction Control of 4WD Vehicles," 12th International Technical Conference on Experimental Safety Vehicles, Sweden, 1989, pp.853-859. 
Table 1 Vehicle parameters

\begin{tabular}{|c|c|c|c|c|}
\hline parameters & $\mathrm{a}$ (meter) & $\mathrm{b}$ (meter) & $\mathrm{h}$ (meter) & $\mathrm{m}(\mathrm{kg})$ \\
\hline values & 1.1 & 1.4 & 0.5 & 1550 \\
\hline
\end{tabular}

